\title{
Clarithromycin for 2 Weeks for Stable Coronary Heart Disease: 6-Year Follow-Up of the CLARICOR Randomized Trial and Updated Meta-Analysis of Antibiotics for Coronary Heart Disease
}

\author{
Christian Gluud $^{\mathrm{a}, \mathrm{b}}$ Bodil Als-Nielsen ${ }^{c}$ Morten Damgaard ${ }^{\mathrm{d}}$ Jørgen Fischer Hansen ${ }^{\mathrm{d}}$ \\ Stig Hansen ${ }^{\mathrm{e}}$ Olav H. Hel $\varnothing^{f}$ Per Hildebrandt $^{g}$ Jørgen Hilden ${ }^{\mathrm{h}}$ Gorm Boje Jensen $^{\mathrm{e}}$ \\ Jens Kastrup ${ }^{f}$ Hans Jørn Kolmos ${ }^{i}$ Erik Kjøller ${ }^{j}$ Inga Lind ${ }^{k}$ Henrik Nielsen ${ }^{c}$ Lars Petersen $^{g}$ \\ Christian M. Jespersen $^{\mathrm{a}} \mathrm{d}^{\mathrm{d}}$ CLARICOR Trial Group ${ }^{1}$

\begin{abstract}
${ }^{a}$ The Copenhagen Trial Unit, Center for Clinical Intervention Research, Rigshospitalet, Copenhagen University Hospital,
${ }^{b}$ The Copenhagen Trial Unit, Center for Clinical Intervention Research, Institute of Preventive Medicine,

'Department of Cardiology, Amager Hospital, Copenhagen University Hospital and d Department of Cardiology Y, Bispebjerg Hospital, Copenhagen University Hospital, Copenhagen, e Department of Cardiology, Hvidovre Hospital, Copenhagen University Hospital, Hvidovre, ${ }^{\mathrm{f}}$ The Heart Center, Department of Medicine B, Rigshospitalet, Copenhagen University Hospital, Copenhagen, ${ }^{9}$ Department of Cardiology E, Frederiksberg Hospital, Copenhagen University Hospital, Frederiksberg, h' Department of Biostatistics, Institute of Public Health, Faculty of Health Sciences, University of Copenhagen, Copenhagen, 'Department of Clinical Microbiology, Odense University Hospital, Odense, 'Department of Cardiology S, Herlev Hospital, Copenhagen University Hospital, Herlev, and kStatens Serum Institut, Copenhagen, Denmark
\end{abstract}

For editorial comment see p. 277

\section{Key Words}

Coronary heart disease - Clarithromycin - Macrolides •

Antibiotics • Cardiovascular mortality $\cdot$ Chlamydia

pneumoniae

\begin{abstract}
Objectives: We have reported increased 2.6-year mortality in clarithromycin- versus placebo-exposed stable coronary heart disease patients, but meta-analysis of randomized trials in coronary heart disease patients showed no significant effect of antibiotics on mortality. Here we report the 6-year mortality of clarithromycin- versus placebo-exposed pa-
\end{abstract}

tients and updated meta-analyses. Methods: Centrally randomized, placebo controlled multicenter trial. All parties were blinded. Analyses were by intention to treat. Metaanalyses followed the Cochrane Collaboration methodology. Results: We randomized 4,372 patients with stable coronary heart disease to clarithromycin $500 \mathrm{mg}(\mathrm{n}=2,172)$ or placebo $(n=2,200)$ once daily for 2 weeks. Mortality was followed through public register. Nine hundred and twentythree patients (21.1\%) died. Six-year mortality was significantly higher in the clarithromycin group (hazard ratio 1.21, 95\% confidence interval 1.06-1.38). Adjustment for entry characteristics (sex, age, prior myocardial infarction, center, and smoking) did not change the results $(1.18,1.04-1.35)$. Ad-

\footnotetext{
1 Members are listed in the Appendix.
}

\begin{tabular}{ll}
\hline KARGER & ( ) 2008 S. Karger AG, Basel \\
0008-6312/08/1114-0280\$24.50/0 \\
$\begin{array}{l}\text { E-Mail karger@karger.ch } \\
\text { www.karger.com }\end{array}$ & $\begin{array}{l}\text { Accessible online at: } \\
\text { www.karger.com/crd }\end{array}$
\end{tabular}

Christian Gluud, MD, Dr Med Sci

The Copenhagen Trial Unit, Center for Clinical Intervention Research

Rigshospitalet, Copenhagen University Hospital

DK-2100 Copenhagen (Denmark)

Tel. +45 354571 75, Fax +45354571 01, E-Mail cgluud@ctu.rh.dk 
dition of our data to that of other randomized trials on antibiotics for patients with coronary heart disease versus placebo/no intervention (17 trials, 25,271 patients, 1,877 deaths) showed a significantly increased relative risk of death from antibiotics of 1.10 (1.01-1.20) without heterogeneity. Conclusions: Our results stress the necessity to consider carefully the strength of the indication before administering antibiotics to patients with coronary heart disease.

Copyright $\odot 2008$ S. Karger AG, Basel

\section{Introduction}

The idea that bacterial colonization of arteries worsened atherosclerosis gained increasing support during the 1990s and several antibiotic trials were launched [1]. Most of these trials were meta-analyzed in 2005 by Andraws et al. [1]. They found no significant effect of antibiotics on all-cause mortality (odds ratio 1.02, 95\% confidence interval $0.89-1.16$ ), but the confidence interval could not exclude an $11 \%$ decrease or a $16 \%$ increase in mortality [1].

We recently reported significantly increased mortality in clarithromycin-exposed patients with stable coronary heart disease [2]. The 2.6-year mortality was $9.8 \%$ in the clarithromycin group compared with $7.8 \%$ in the placebo group (hazard ratio 1.27, 1.03-1.54). When we added these data [2] and data from the trial of Berg et al. [3] to the meta-analysis of Andraws et al. [1], we found no significant effect of antibiotics across dosages, regimens, and duration of follow-up on mortality of coronary heart disease patients (odds ratio 1.09, 0.97-1.22) [2]. The confidence interval could not exclude a $3 \%$ decrease or a $22 \%$ increase in mortality [2]. When we pooled the data of three trials following patients for more than 2 years, i.e., the CLARICOR, PROVE-IT, and ACES trials $[2,4,5]$, antibiotics were associated with a significantly increased mortality $(1.20,1.04-1.39)$ [2].

To examine the association between short-term clarithromycin and long-term mortality, we did an extended follow-up of the CLARICOR patients. Further, we updated our meta-analyses to examine the effect of antibiotics on mortality in patients with coronary heart disease.

\section{Methods}

CLARICOR is a randomized, placebo-controlled, blinded, parallel-group multicenter trial in patients with stable coronary heart disease $[2,6]$. The Copenhagen Trial Unit handled all administrative functions, including centralized randomization, communication with blinded outcome assessors, and contact with database managers. The patients were enrolled at five academic cardiology departments in Copenhagen.

The central hospital database of Copenhagen enabled us to identify all patients with a diagnosis of myocardial infarction and/or angina pectoris (ICD 209-219) during the years 19931999. Patients, aged 18-85 years, were invited for an interview at one of the five cardiology units. The exclusion criteria have previously been described $[2,6]$.

Ethical approval was given by the local ethics committee (KF 01-076/99), the Danish Medicines Agency (2612-975), and The Danish Data Protection Agency (1999-1200-174).

Randomization and Interventions

Eligible patients were stratified according to sex, prior myocardial infarction, age below 60 years, and center and were randomly assigned to oral clarithromycin (Klacid Uno ${ }^{\circledR}$, Abbott, UK) $500 \mathrm{mg}$ or matching placebo once daily for 2 weeks $[2,6]$. Four thousand three hundred and seventy-two patients were randomized between October 5, 1999 and April 15, 2000: 2,172 to clarithromycin and 2,200 to placebo. The intervention groups were well matched with the exception of smoking habit [2].

Treatment Diary, Outcomes, and Follow-Up

More than $90 \%$ of the patients in each arm reported taking at least 13/14 tablets prescribed during the trial [2]. Clarithromycin was well tolerated during and just after its administration [2]. A blinded search of the Danish Central Civil Register records for the vital status of the participants was carried out on December 5, 2005. The Register has almost $100 \%$ validity. The search more than doubled our observation time and forms the basis of the extended follow-up. The participants that were still alive were informed about the results of the trial.

Statistical Analyses and Meta-Analyses

SAS statistical package version 6.12-8 was used for the statistical analyses. The hazard ratios and their confidence intervals are based on a Cox regression model, which according to the protocol included sex, prior myocardial infarction, age, and center as covariates. All covariates not well matched at randomization (i.e., smoking) [2] and information on medication and diabetes at entry holding prognostic information were introduced in exploratory analyses. Event curves were constructed according to the Kaplan-Meier method.

We updated our previous meta-analyses [2] with data from the extended follow-up of CLARICOR patients as well as data from new trials identified in January 2007 through searches in The Cochrane Library, PubMed, and EMBASE according to our protocol [7]. Meta-analyses followed the Cochrane Collaboration methodology [7], including Review Manager [8] for calculation of relative risks with 95\% CI with random-effect models and inconsistency $\left(I^{2}\right)$ as a measure of heterogeneity [9].

\section{Results}

The mean follow-up from randomization was 5.9 years (range 5.7-6.2 years). In the clarithromycin group 13 patients $(0.6 \%)$ and in the placebo group 15 patients $(0.7 \%)$ were censored due to emigration or disappearance. Of 


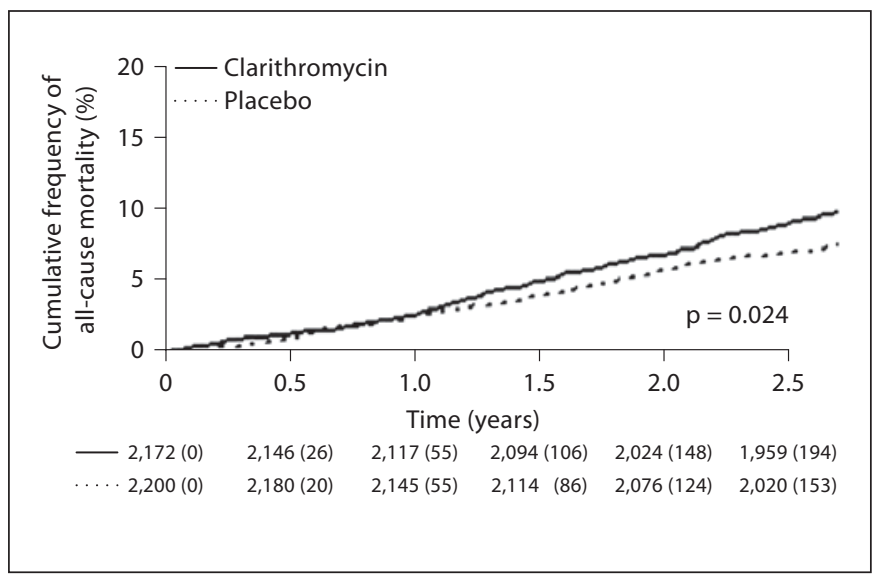

Fig. 1. Unadjusted Kaplan-Meier estimates of all-cause cumulative mortality in the clarithromycin group and in the placebo group during 2.6 years of follow-up. Figures below the diagram are the number of participants still at risk (followed by the cumulative number of deaths) in the clarithromycin and placebo groups.

the remaining $99.4 \%$ of the patients, survival status was known.

\section{All-Cause Mortality}

As of December 5, 2005, 923/4,372 (21.1\%) patients had died. The number of deaths among clarithromycin patients was 497/2,172 (22.9\%) compared with 426/2,200 (19.4\%) among placebo patients. This difference is significant during the first 2.6-year follow-up (univariate hazard ratio $1.26,1.03-1.54, \mathrm{p}=0.024$ ) (fig. 1) and during the 6-year follow-up (univariate hazard ratio 1.21, 1.06$1.38, \mathrm{p}=0.004$ ) (fig. 2). The excess mortality was uniform over time (fig. 3). The increased mortality corresponds to one death during 6 years of follow-up out of 22 patients (13-70 patients) treated with clarithromycin.

Table 1 shows the number of patients dying in the clarithromycin group and the placebo group stratified according to sex, age, prior myocardial infarction, diabetes, and smoking habits at entry. In all subgroups, the proportion of patients dying in the clarithromycin group was larger than in the placebo group.

\section{Multivariate Analyses}

The increased mortality risk in the clarithromycin group persists in the multivariate analysis including sex, age, prior myocardial infarction, and center as covariates $(1.20,1.06-1.37, \mathrm{p}=0.0048)$. When smoking status at entry was added as an additional covariate, the increased mortality risk in the clarithromycin group also

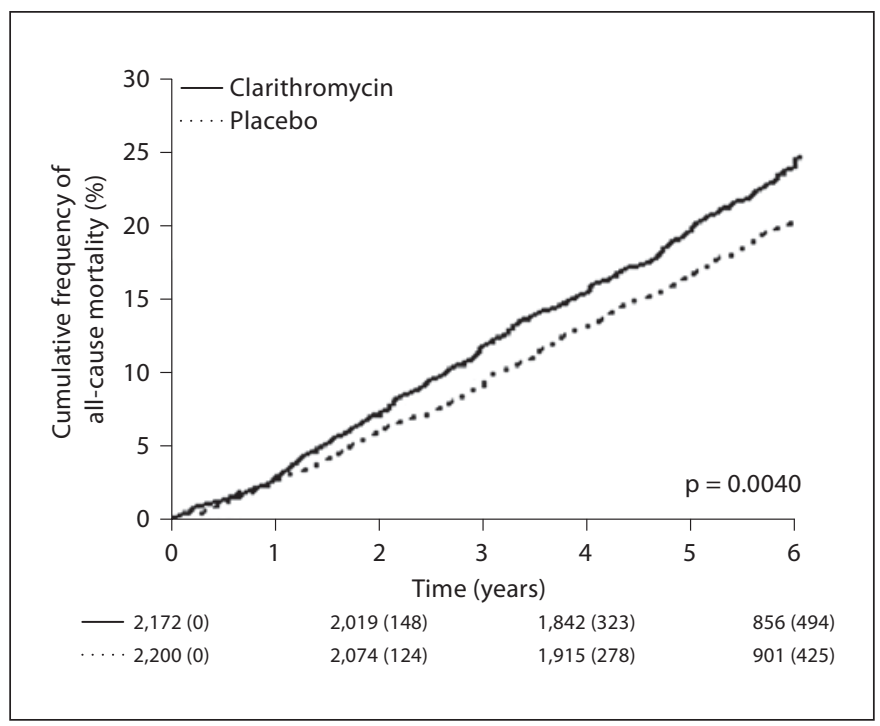

Fig. 2. Unadjusted Kaplan-Meier estimates of all-cause cumulative mortality in the clarithromycin group and in the placebo group during 6 years of follow-up. Figures below the diagram are the number of participants still at risk (followed by the cumulative number of deaths) in the clarithromycin and placebo groups. Until 5.7 years after randomization the graph is based on the full cohorts.

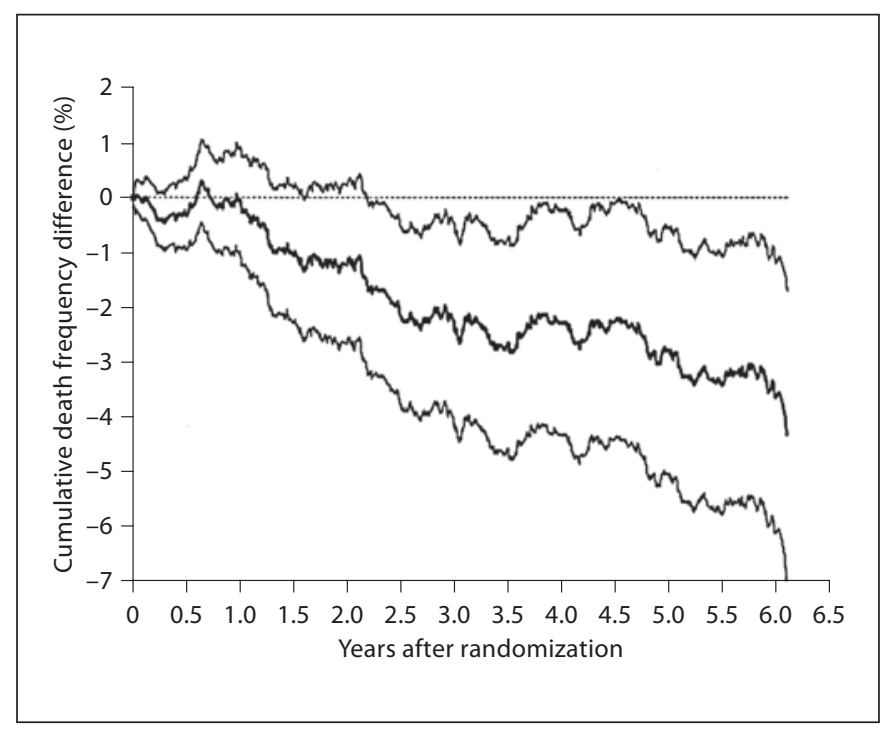

Fig. 3. Cumulative difference in the estimated all-cause risk of death during 6 years of follow-up, with $95 \%$ confidence limits. A negative value indicates that placebo patients did better than clarithromycin patients. Until 5.7 years after randomization the graph is based on the full cohorts. The number of deaths and the number at risk are as shown in figure 2. 
Table 1. Six-year all-cause mortality in subgroups of patients with stable coronary heart disease treated with clarithromycin or placebo

\begin{tabular}{lcc}
\hline Subgroup & Clarithromycin & Placebo \\
\hline Sex & & \\
$\quad$ Female & $148 / 658(22.4)$ & $122 / 681(17.9)$ \\
$\quad$ Male & $349 / 1,514(23.0)$ & $304 / 1,519(20.1)$ \\
Age & & \\
$\quad$ Below 60 years & $70 / 640(10.9)$ & $45 / 663(6.7)$ \\
$\quad$ At or above 60 years & $427 / 1,532(27.8)$ & $381 / 1,537(24.7)$ \\
Prior myocardial infarction & & \\
$\quad$ No & $124 / 702(17.6)$ & $123 / 706(17.4)$ \\
$\quad$ Yes & $373 / 1,470(25.3)$ & $303 / 1,494(20.2)$ \\
Diabetes mellitus 1 & & \\
$\quad$ No & $405 / 1,831(22.1)$ & $341 / 1,863(18.3)$ \\
$\quad$ Yes & $92 / 341(26.9)$ & $85 / 337(25.2)$ \\
Smoking status & & \\
$\quad$ Never & $65 / 372(17.5)$ & $66 / 435(15.2)$ \\
$\quad$ Previous & $218 / 981(22.2)$ & $192 / 1,012(18.9)$ \\
$\quad$ Current & $214 / 819(26.1)$ & $168 / 753(22.3)$ \\
\hline
\end{tabular}

${ }^{1}$ One surviving placebo-treated patient lacked information on diabetes status and is included in the no diabetes mellitus group.
$0.02)$ without heterogeneity $\left(I^{2}=0 \%\right)$, favoring placebo or no intervention (fig. 4).

We found no significant difference between our CLARICOR mortality data $(1.18,1.05-1.33)$ compared to those of the other 16 trials $(1.02,0.90-1.16)$ in a test of interaction $(\mathrm{z}=1.65$, nonsignificant).

Two other trials on clarithromycin for coronary heart disease patients have been published [3,15]. Meta-analyzing these data with our present data shows a statistically significant increased mortality in the clarithromycin group compared with the placebo group $(1.19,1.06-1.33$, $\mathrm{p}=0.004)$ without heterogeneity $\left(I^{2}=0 \%\right)$ (data not shown). The intervention effect of clarithromycin in these trials seemed more pronounced than in the remaining 14 trials on other antibiotics $(1.01,0.89-1.15)$, but the difference was not significant $(\mathrm{z}=1.78)$.

Meta-analysis of the three trials that followed patients for more than 2 years, i.e., PROVE-IT [4], ACES [5], and CLARICOR, showed that antibiotics are associated with significantly increased mortality $(1.17,1.06-$ $1.29, \mathrm{p}=0.002)$ without heterogeneity $\left(I^{2}=0 \%\right)($ data not shown).

\section{Discussion}

persists $(1.18,1.04-1.35, \mathrm{p}=0.0108)$. We observed similar odds ratios for death comparing clarithromycin versus placebo in never-smokers, ex-smokers, and current smokers. Further analyses demonstrated that only few of the extra deaths in the clarithromycin group could be ascribed to smoking (data not shown). The hazard ratio remains stable when additional adjustments were made for the following eight binary entry covariates: use of digoxin; diuretics; ACE inhibitors; antiarrhythmics; longacting nitrates and diabetes (all associated with poor prognosis), and use of acetylsalicylic acid and statins (both associated with favorable prognosis) $(1.18,1.03-$ $1.35, \mathrm{p}=0.015)$.

\section{Meta-Analyses}

Figure 4 shows the updated meta-analysis including data from the 11 randomized trials from the meta-analysis of Andraws et al. [1] and the Berg et al. [3], ISAR-3 [10], Parachure et al. [11], Brown et al. [12], Radoi et al. [13], and CLARICOR trials. We were unable to obtain mortality data from one trial including 141 patients [14].

The 17 randomized trials randomized 12,643 patients to antibiotics and 12,628 patients to placebo or no intervention. The number of deaths was 1,877 . The corresponding overall relative risk ratio is $1.10(1.01-1.20, \mathrm{p}=$
According to our findings, short-term intervention with clarithromycin seems to be associated with a significantly increased long-term mortality in patients with stable coronary heart disease. Our findings persist after multivariate adjustment. Furthermore, our findings are supported by analyses of subgroups, which all had higher mortality in the clarithromycin group compared with the placebo group. The results of our updated meta-analyses may potentially incriminate all antibiotics given to patients with stable coronary heart disease and acute coronary syndromes. Clarithromycin (or potentially antibiotics at large) may cause the detrimental effect by interfering with the host-parasite balance. Chlamydia pneumoniae replicate in monocytes in atherosclerotic lesions and secure their local persistence by inhibiting host cell apoptosis [16]. A short clarithromycin course may not eradicate $C$. pneumoniae from the monocytes $[17,18]$. On the contrary, clarithromycin may induce apoptosis of infected monocytes [19-21]. This could lead to enhanced release of viable Chlamydia into the bloodstream and dissemination of infection. This may increase inflammation and aggravate the cardiovascular disease, which eventually leads to death. The reason why this detrimental effect has not been noticed 


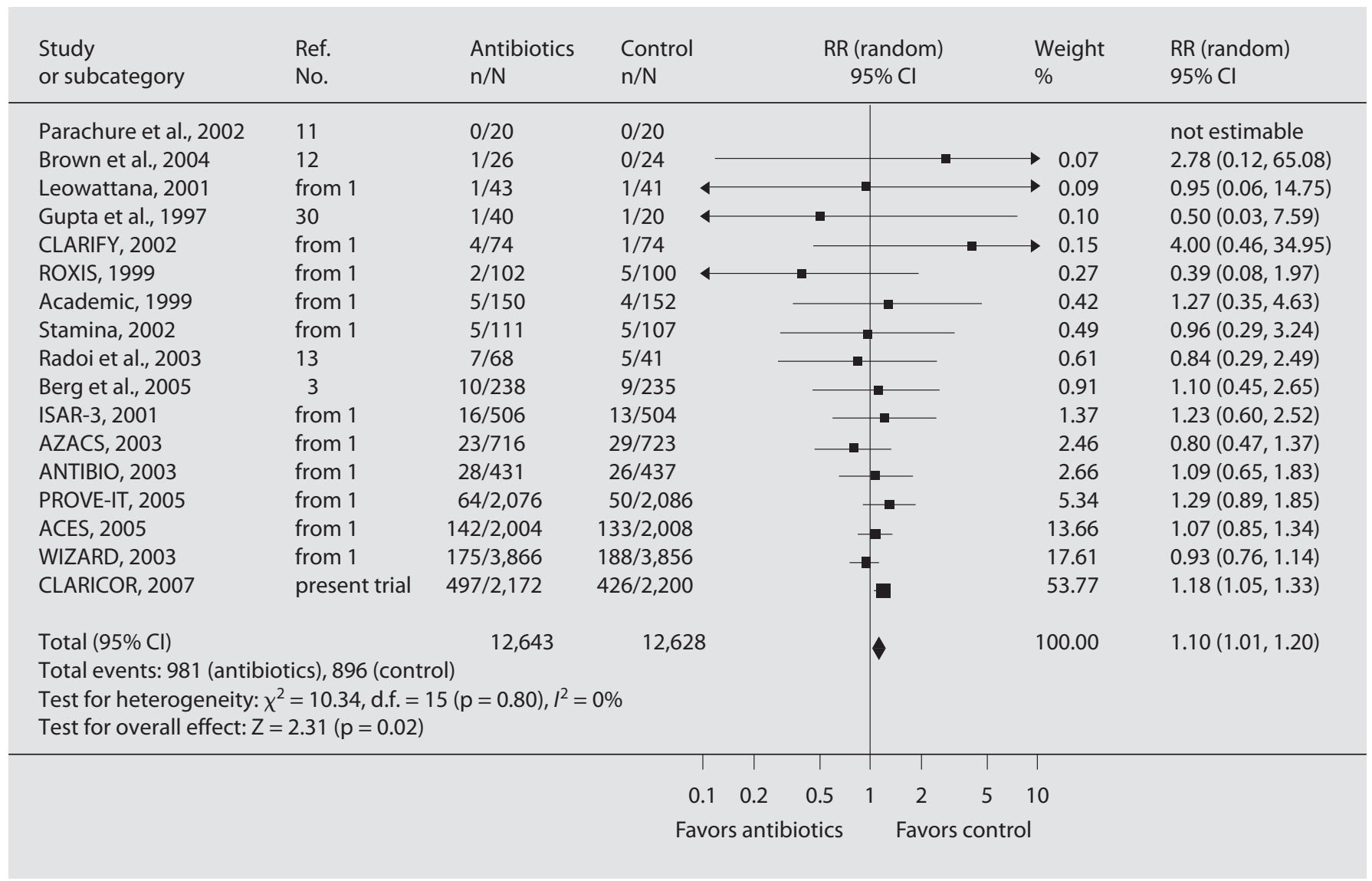

Fig. 4. Intervention effect of different antibiotics versus placebo or no intervention on mortality of patients with coronary heart disease. $\mathrm{CI}=$ Confidence interval; $\mathrm{n}=$ number of patients with outcome; $\mathrm{N}$ = number of participants at risk; d.f. = degrees of freedom; $I^{2}=$ percentage of total variation across studies that is due to heterogeneity rather than chance. Relative risks are plotted (black squares with area proportional to the amount of statistical information in each trial) comparing outcome among partici- pants allocated to antibiotic with those allocated to placebo or no intervention, alongside their 95\% CI (horizontal lines). For totals, the result and its 95\% CI are represented by a diamond with the relative risk and 95\% CI given alongside. Squares or diamonds to the right of the solid vertical line indicate harm from antibiotics. This is conventionally significant $(\mathrm{p}<0.05)$ only if the horizontal line or diamond does not overlap the solid vertical line. may be due to too short a follow-up time in previous randomized trials.

\section{Strengths and Limitations}

Our trial offers several strengths, including its size, the long follow-up time, and the high number of outcomes. These components should reduce random errors. One may get the impression that it takes 12-24 months before the intervention curves depart. In fact, we observed a constant increase in mortality in the clarithromycintreated patients, with minimal fluctuation during the first 18 months. This can be ascribed to too few outcomes occurring during this period. The central randomization, the placebo-controlled intervention coupled with blinded outcome assessment, and intention-to-treat analyses [2] should have reduced systematic errors [22-24]. Furthermore, follow-up was via public registers, ensuring few losses. We looked exclusively at all-cause mortality, which should not carry the interpretative difficulties that are often encountered with cardiovascular composite outcomes [25].

Even if we adjust our univariate $\mathrm{p}=0.004$ for previous analyses of our primary, secondary, and tertiary outcomes as well as our previous mortality analysis, we find a significant detrimental effect of clarithromycin. However, we cannot of course exclude the possibility of random errors. For completeness, further information on physical and medical status at randomization and chang- 
es in medical treatment or life style during follow-up could have been sought. However, we find it unlikely that these factors should differ substantially in the two intervention groups. The only difference recorded at entry was a small difference in smoking status [2]. Taking this difference into consideration did not modify our results noticeably. In fact, the overrepresentation of current smokers in the clarithromycin group could only account for some few extra deaths and we observed very similar relative risk ratios of clarithromycin versus placebo in the subgroups of never-smokers, ex-smokers, and current smokers (table 1). When one factor is not fully balanced, other unaccounted factors could be out of balance. We cannot exclude this possibility, but at least four arguments speak against it. We found no inconsistency among the trials of our meta-analyses. $I^{2}$ was $0 \% . I^{2}$ is the percentage of between-trial variability that is due to true differences between trials (heterogeneity) rather than sampling error (chance). The estimates of intervention effect in the CLARICOR trial or the three clarithromycin trials were not significantly different from that observed in meta-analyses of the remaining trials. Our results are in accordance with the results of other antibiotic trials with long-term follow-up.

\section{Comparison with Related Research}

Several randomized trials on clarithromycin, azithromycin, roxithromycin, spiramycin, gatifloxacin, or doxycycline for coronary heart disease patients have been published [1-3, 10-15]. Our meta-analysis shows that antibiotics irrespective of type, duration of treatment, dose, and duration of follow-up are associated with a significantly increased mortality. This effect was seen both in patients with stable coronary heart disease and in patients with acute coronary syndromes, although it only reached formal statistical significance in the former (data not shown). However, the trials with acute coronary syndrome patients were generally small and had a short follow-up time.

Duration of follow-up is critical. If we had stopped follow-up within 2 years, we could have had an insignificant result as most other trials did. If we pool the data of the three trials following patients for more than 2 years, antibiotics are associated with significantly increased mortality. The CLARICOR trial randomized less than 19\% of the patients in the meta-analyses including all trials, but carries more than $53 \%$ of the weight due to the high occurrence of outcomes.

Our previously blinded adjudication of 384 deaths identified cardiovascular causes as responsible for the increased mortality [2]. This increases the likelihood that the observed association is causal compared to a finding of a diffuse increase in all causes of mortality. Macrolides possess potassium channel blocker properties, which may increase the risk of torsades de pointes tachycardia and sudden death [26]. We observed no differences in cardiovascular mortality during the treatment itself or during the first months of follow-up [2]. As stressed above, the excess mortality was uniform over time and we found no significant association between time and the increase in mortality. As suggested, antibiotics might interfere unfavorably with the host-parasite balance in atherosclerotic lesions and result in thrombotic events. The US FDA has reacted with concern to our trial result [27], and so has the EU EMEA. The Danish Medicines Agency, however, found no reason for concern [28]. They conducted a prescription study of patients on regular acetylsalicylic acid, comparing clarithromycin to other antibiotics [28]. The Danish Medicines Agency found clarithromycin to be significantly less associated with mortality than other antibiotics [28]. The findings may be interpreted as inculpating several types of antibiotics.

\section{Conclusions}

A short clarithromycin course for patients with stable coronary heart disease was associated with significantly ( $p=0.004)$ more deaths during long-term follow-up of patients living in Copenhagen. Updated meta-analyses of randomized trials suggest that this detrimental effect may involve antibiotics at large $(p=0.02)$. These $p$ values are too small to be ignored and more importantly so is the direction of the effect of antibiotics. At the same time, the $p$ values are not small enough to completely exclude the risk of random errors. Accordingly, more data are needed. Until such data have been scrutinized we urge the public and health care workers to consider that we have observed the detrimental effect among patients who are not normally prescribed antibiotics unless they demonstrate signs of active infection. The potential negative consequences of antibiotics based on the present findings should therefore be weighted against any benefits that may be obtained when treating life-threatening infections in such patients with antibiotics. We therefore suggest that our results should be considered when clarithromycin and other antibiotics are prescribed for patients with coronary heart disease. Moreover, we suggest action by regulatory authorities. We urgently need longer follow-up results from the many randomized trials conducted. 
Antibiotics for patients with coronary heart disease may be yet another intervention area [29] where initial small positive trials $[30,31]$ led to false hopes, numerous large trials, and negative conclusions $[32,33]$. The initial trials also generated a plethora of proposed disease mechanisms. Only future studies can tell if too little or too much antibiotic has been used $[32,34]$. The links between antibiotics, inflammation, and atherothrombosis may be more complicated than previously perceived [34].

\section{Acknowledgments}

We thank The Danish Heart Foundation, The Copenhagen Hospital Corporation, The Danish Research Council, and the 1991 Pharmacy Foundation for unrestricted grants. The clarithromycin and placebo tablets were supplied by Abbott Laboratories, IDC, Queensborough, UK. We thank Jørn Wetterslev for help during the inspection of the CLARICOR Trial. We thank Mariana Radoi and Ioana Agasche for providing us with 4-year mortality data from their randomized trial.

Funding: The CLARICOR Trial is investigator initiated and controlled. This work was supported by grants from nonprofit funds (The Danish Heart Foundation, The Copenhagen Hospital Corporation, The Danish Research Council, and The 1991 Pharmacy Foundation). Abbott Laboratories, IDC, Queensborough, UK supplied the clarithromycin and placebo tablets. Those supporting the trial had no role in design, data collection, data analyses, data interpretation, or writing the report. The Steering Group had full access to all the data and had final responsibility for the decision to submit the report for publication.

\section{Appendix}

Contributors: $\mathrm{HJK}$ had the original idea for the trial. The Steering Committee consisted of CG (chair), JFH, PH, GBJ, JK, HJK, IL, $\mathrm{HN}$, and CMJ. CG, JFH, PH, GBJ, JK, IL, HN, and CMJ were principle investigators. BA-N, MD, LP, SH, and $\mathrm{OHH}$ were clinical investigators. CG and $C M J$ were coordinating investigators. $C G$ and CMJ drafted the protocol. CG and JH drafted this report. CG is guarantor. EK was chairman of the Event Committee. JH was member of the independent Data Monitoring and Safety Committee and conducted the statistical survival analyses assisted by Per Jensen and Christian Pipper. CG conducted the meta-analyses. Of the contributors who are not listed as authors Jette Mieritz (Amager Hospital), Lene Thuesen (Bispebjerg Hospital), Hanne Larsen, Per Nielsen, Merethe Hildebrandt (Frederiksberg Hospital), Mette Bommersholdt (Hvidovre Hospital), and Lone Kristensen (Rigshospitalet) were study nurses. Jette V Petersen and Pia Hughes ${ }^{\dagger}$ coordinated the trial data accrual and data entry. Bitten Hansen, Ninna Frydendahl, Bessie Hødholdt, Karen Juliussen, and Mette Hansen executed data accrual. Kjell Nillson and Nader Salas developed the randomization system and the data management systems. Nader Salas was responsible for data management. The independent Data Monitoring and Safety Committee was chaired by Kristian Thygesen (Århus, Denmark) with John Kjekshus (Oslo, Norway) and JH (Copenhagen, Denmark) as members. Stig Haun$s ø$ (Rigshospitalet, Copenhagen, Denmark), Rolf Steffensen (KAS Gentofte, Hellerup, Denmark), and Thorkild I.A. Sørensen (Institute of Preventive Medicine, Copenhagen, Denmark) advised the Steering Group during the planning of the trial.

\section{References}

1 Andraws R, Berger JS, Brown DL: Effects of antibiotic therapy on outcomes of patients with coronary artery disease: a meta-analysis of randomized controlled trials. JAMA 2005;293:2641-2647.

-2 Jespersen CM, Als-Nielsen B, Damgaard M, Hansen JF, Hansen S, Helø OH, et al: Randomised placebo controlled multicentre trial to assess short term clarithromycin for patients with stable coronary heart disease: CLARICOR trial. BMJ 2006;332:22-27. DOI: 10.1136/bmj.38666.653600.55.

- 3 Berg HF, Maraha B, Scheffer G-J, Quarles van Ufford M, Vandenbroucke-Grauls CM, Peeters MF, Kluytmans JA: Treatment with clarithromycin prior to coronary artery bypass graft surgery does not prevent subsequent cardiac events. Clin Infect Dis 2005; 40:358-365.

4 Cannon CP, Braunwald E, McCabe CH, Grayston JT, Muhlestein B, Giugliano RP, et al: Antibiotic treatment of Chlamydia pneumoniae after acute coronary syndrome. $\mathrm{N}$ Engl J Med 2005;352:1646-1654.
5 Grayston JT, Kronmal R, Jackson LA, Parisi AF, Muhlestein JB, Cohen JD, et al: Azithromycin for the secondary prevention of coronary events. N Engl J Med 2005;352:16371645.

-6 Hansen S, Als-Nielsen B, Damgaard M, Helø $\mathrm{OH}$, Petersen L, Jespersen CM, The CLARICOR Group: Intervention with clarithromycin in patients with stable coronary heart disease: the CLARICOR trial design. Heart Drug 2001;1:14-19.

7 Als-Nielsen B, Gluud C: Antibiotics for secondary prevention of coronary heart disease (protocol). Cochrane Database Syst Rev 2002;2:CD003610. DOI: 10.1002/14651858. CD003610.

8 Review Manager (RevMan) (Computer Program). Version 4.2 for Windows. Copenhagen, The Nordic Cochrane Centre, The Cochrane Collaboration, 2003.

-9 Higgins JPT, Thompson SG: Quantifying heterogeneity in a meta-analysis. Stat Med 2002;21:1539-1558.
10 Neumann F-J, Kastrati A, Miethke T, Pogatsa-Murray G, Mehilli J, Valina C, et al: Treatment of Chlamydia pneumoniae infection with roxithromycin and effect on neointima proliferation after coronary stent placement (ISAR-3): a randomised, double-blind, placebo-controlled trial. Lancet 2001;357:20852089.

11 Parachure N, Zouridakis EG, Kaski JC: Effect of azithromycin treatment on endothelial function in patients with coronary artery disease and evidence of Chlamydia pneumoniae infection. Circulation 2002;105: 1298-1303.

12 Brown DL, Desai KK, Vakili BA, Nouneh C, Lee HM, Golub LM: Clinical and biochemical results of the metalloproteinase inhibition with subantimicrobial doses of doxycycline to prevent acute coronary syndromes (MIDAS) pilot trial. Arterioscler Thromb Vasc Biol 2004;24:733-738.

13 Radoi M, Bobescu E, Agache I: Rovamycine as add-on treatment in unstable angina and 4 year evolution with major cardiovasular events. Rom J Intern Med 2003;41:237-246. 
14 Hillis GS, Pearson CV, Harding SA, Sutherland S, Ludlam CA, Marioni JC, et al: Effects of a brief course of azithromyzin on soluble cell adhesion molecules and markers of inflammation in survivors of an acute coronary syndrome: a double-blind, randomized, placebo-controlled study. Am Heart J 2004;148:72-79.

-15 Sinisalo J, Mattila K, Valtonen V, Anttonen $\mathrm{O}$, Juvonene J, Melin J, et al: Effect of 3 months of antimicrobial treatment with clarithromycin in acute on $\mathrm{Q}$ wave coronary syndrome. Circulation 2002;105:1555-1560.

-16 Fischer SF, Vier J, Kirschnek S, Klos A, Hess S, Ying S, et al: Chlamydia inhibits host cell apoptosis by degradation of proapoptotic BH3-only proteins. J Exp Med 2004;200: 905-916.

17 Gieffers J, Füllgraf H, Jahn J, Klinger M, Dalhoff K, Katus HA, et al: Chlamydia pneumoniae infection in circulating human monocytes is refractory to antibiotic treatment. Circulation 2001;103:351-356.

18 Berg HF, Maraha B, Scheffer G-J, Peeters MF, Kluytmans JAJW: Effect of clarithromycin on inflammatory markers in patients with atherosclerosis. Clin Diagn Lab Immunol 2003; 10:525-528.

19 Ohara T, Kanoh Y, Higuchi K, Arakawa T, Morisita T: Eradication therapy of Helicobacter pylori directly induces apoptosis in inflammation-related immunocytes in the gastric mucosa - possible mechanism for cure of peptic ulcer disease and MALT lymphoma with low-grade malignancy. Hepatogastroenterology 2003;50:607-609.
20 Mizunoe S, Kadota J, Tokimatsu I, Kishi K, Nagai H, Nasu M: Clarithromycin and azithromycin induce apoptosis of activated lymphocytes via down-regulation of $\mathrm{Bcl}-\mathrm{xL}$. Int Immunopharmacol 2004;4:1201-1207.

21 Ishimatsu Y, Kadota J, Iwashita T, Nagata T, Ishii H, Shikuwa C, et al: Macrolide antibiotics induce apoptosis of human peripheral lymphocytes in vitro. Int $J$ Antimicrob Agents 2004;24:49-55.

22 Schulz KF, Chalmers I, Hayes RJ, Altman DG: Empirical evidence of bias: dimensions of methodological quality associated with estimates of treatment effects in controlled trials. JAMA 1995;273:408-412.

23 Moher D, Pham B, Jones A, Cook DJ, Jadad AR, Moher M, et al: Does quality of reports of randomised trials affect estimates of intervention efficacy reported in meta-analyses? Lancet 1998;352:609-613.

24 Kjærgard LL, Villumsen J, Gluud C: Reported methodological quality and discrepancies between large and small randomized trials in meta-analyses. Ann Intern Med 2001;135: 982-989.

25 Ferreira-González I, Busse JW, Heels-Ansdell D, Montori VM, Akl EA, Bryant DM, et al: Problems with use of composite end points in cardiovascular trials: systematic review of randomised controlled trials. BMJ 2007;334:786. DOI: 10.1136/ bmi.39136.682083.AE.

26 Martin D, Bursill J, Qui MR, Breit SN, Campbell T: Alternative hypothesis for efficacy of macrolides in acute coronary syndromes. Lancet 1998;351:1858-1859.
27 Food and Drug Agency: FDA Alert for Healthcare Professionals: Clarithromycin (in the CLARICOR Study). http://www.fda. gov/cder/drug/InfoSheets/HCP/clarithromycinHCP.pdf (accessed November 14, 2007)

28 The Danish Medicines Agency: Clarithromycin for patients with ischemic heart disease. December 8, 2005. http://www.dkma. $\mathrm{dk} / 1024 /$ visUKLSArtikel.asp?artikelID= 7597 (accessed November 14, 2007).

29 Ioannidis JP: Contradicted and initially stronger effects in highly cited clinical research. JAMA 2005;294:218-228.

-30 Gupta S, Leatham EW, Carrington D, Mendall MA, Kaski JC, Camm AJ: Elevated Chlamydia pneumoniae antibodies, cardiovascular events, and azithromyzin in male survivors of myocardial infarction. Circulation 1997;96:404-407.

31 Gurfinkel E, Bozovich G, Daroca A, Beck E, Mautner B, ROXIS Study Group: Randomised trial of roxithromycin in non- $Q$ wave coronary syndromes: ROXIS pilot study. Lancet 1997;350:404-407.

32 Grayston JT: Antibiotic treatment of atherosclerotic cardiovascular disease. Circulation 2003;107:1228-1230.

33 Bjelakovic G, Nikolova D, Gluud LL, Simonetti RG, Gluud C: Mortality in randomized trials of antioxidant supplements for primary and secondary prevention. Systematic review and meta-analysis. JAMA 2007;297: 842-857.

34 Libby P, Ridker PM: Inflammation and atherothrombosis. From population biology and bench research to clinical practice. J Am Coll Cardiol 2006;48(suppl 1):A33-A46. 